\title{
Sex bias in basic and preclinical age-related hearing loss research
}

\author{
Dillan F. Villavisanis ${ }^{*}$ (D, Katrina M. Schrode and Amanda M. Lauer
}

\begin{abstract}
Objectives: This study aims to determine if there is sex bias in basic and preclinical research on age-related hearing loss for the 10-year period of 2006-2015, prior to the NIH mandate of including sex as a biological variable in 2016.

Design: Manuscripts were identified in PubMed for the query "age-related hearing loss" for the 10-year period of 2006 to 2015. Manuscripts were included if they were original research (not reviews or meta-analyses), written in English, contained an abstract, used animals, and were primarily on age-related hearing loss. These criteria yielded 231 unique manuscripts for inclusion in the study analysis. The text of each manuscript was screened for the sex of the animals, the number of male and female animals, the discussion of sex-based results, the study site (US or international), and the year of publication.

Results: Only two thirds of manuscripts reported the sex of animals used in the experiments, and of these, 54\% used both sexes, 34\% used males only, and 13\% used females only. In papers reporting sex and number of animals used, $67 \%$ were males and 33\% were females. Over twice as many internationally based studies used males only compared to US-based studies. Only 15\% of all manuscripts discussed sex-based results.

Conclusions: Sex bias is present in basic and preclinical age-related hearing loss research for the manuscripts screened in the 10-year period. Equal inclusion of both males and females in basic and preclinical age-related hearing loss research is critical for understanding sex-based differences in mechanisms and for effective treatment options.
\end{abstract}

Keywords: Sex bias, Hearing loss, Basic, Preclinical, Animals, NIH

\section{Background}

Age-related hearing loss (ARHL) or presbycusis is the gradual loss of hearing sensitivity with age. It affects one in three people between the ages of 65 to 74 and nearly half of people over 75 in the USA (National Institute on Deafness and Other Communication Disorders). Since the exact biological causes and mechanisms of ARHL are not completely known [1], preclinical and basic science research has been crucial for informing the current understanding of ARHL through physiological [2], anatomical [3], behavioral [4], and genetic [5] studies. ARHL has clinical associations with cognitive decline [6], social isolation [7], and memory loss [8], as well as increased risk of falls [9], hospitalization [10], and mortality [11], which underscore the importance of preclinical and basic science investigations of this condition. There are well-known differences in the trajectory of ARHL

\footnotetext{
* Correspondence: dvillav1@jhu.edu

Department of Otolaryngology_-Head and Neck Surgery, Center for Hearing and Balance, Johns Hopkins University School of Medicine, 720 Rutland Ave.,
} Baltimore, MD 21205, USA

(c) The Author(s). 2018 Open Access This article is distributed under the terms of the Creative Commons Attribution 4.0 International License (http://creativecommons.org/licenses/by/4.0/), which permits unrestricted use, distribution, and reproduction in any medium, provided you give appropriate credit to the original author(s) and the source, provide a link to the Creative Commons license, and indicate if changes were made. The Creative Commons Public Domain Dedication waiver (http://creativecommons.org/publicdomain/zero/1.0/) applies to the data made available in this article, unless otherwise stated. faster decline in hearing ability than women $[12,13]$.

Using animals in preclinical and basic science research provides a unique advantage in understanding the effects of age on hearing loss since the auditory environment and other potentially interacting factors can be carefully controlled. However, the number of preclinical studies that explicitly investigated the effects of sex-based differences on AHRL is relatively small [14-20], and further investigations are needed for a more comprehensive understanding. In C57BL/6 mice, females demonstrated accelerated increases in auditory brainstem response (ABR) thresholds with age compared to males $[19,20]$. In another study, young male and female CBA mice (substrain unspecified) demonstrated similar distortion product otoacoustic emissions (DPOAEs), but females demonstrated higher premenopausal DPOAE levels, which declined postmenopause. Old CBA females also had lower ABR thresholds than old CBA males [18]. Studies investigating the protective effects of 
exposure to an augmented acoustic environment against ARHL have also shown differences in treatment effectiveness between males and females $[15,16,20]$.

Basic and preclinical research has clear and direct implications for translational research and clinical outcomes [21]. Therefore, an understanding of sex as a biological variable and its effects on preclinical research, and here particularly on ARHL research, is important for informing treatment and prevention strategies. The National Institutes of Health (NIH) has required the inclusion of men and women in NIH-funded clinical research since 1993 through the Revitalization Act, but the inclusion of both sexes in basic and preclinical research was not required by the NIH until January of 2016 [22]. This is significant as NIH-funded basic research contributes substantially to drug development [23], and studies have demonstrated that mammalian traits in both wildtypes and mutants are influenced by sex [24].

Other fields have addressed the issue of sex bias in preclinical and basic research including dermatology [25], cardiology [26], surgery, [27], and general neuroscience $[28,29]$. However, sex bias has only been addressed in one study with a focus on auditory neuroscience that identified sex bias in preclinical and basic noise-induced hearing loss (NIHL) research [30]. In the present study, the representation of sex as a biological variable in basic and preclinical studies of ARHL was evaluated for the 10-year period from January 2006 to December 2015 to understand sex bias in studies of ARHL prior to the NIH mandate.

\section{Materials and methods}

Literature search and inclusion criteria

Manuscripts were identified in PubMed using the query "age-related hearing loss," which generated a total of 4090 unique manuscripts. A parameter for the 10-year period from January 1, 2006, to December 31, 2015, was applied, yielding 1861 manuscripts. Filters were applied to select for manuscripts including an abstract, written in English, and including other animals (non-human), which generated 363 manuscripts. Manuscripts were exported to a Microsoft Excel file and manually screened for exclusion if (1) a majority of the experiments and results were not primarily concerning ARHL $(n=62)$; (2) manuscripts were reviews, meta-analyses, or non-original research $(n=61)$; (3) experiments were not performed in animals $(n=5)$; and (4) had no full text available through PubMed or the Johns Hopkins Libraries $(n=4)$. Following exclusion, a total of 231 manuscripts were included in this study for analysis. Inclusion criteria are depicted in the flow diagram (Fig. 1).

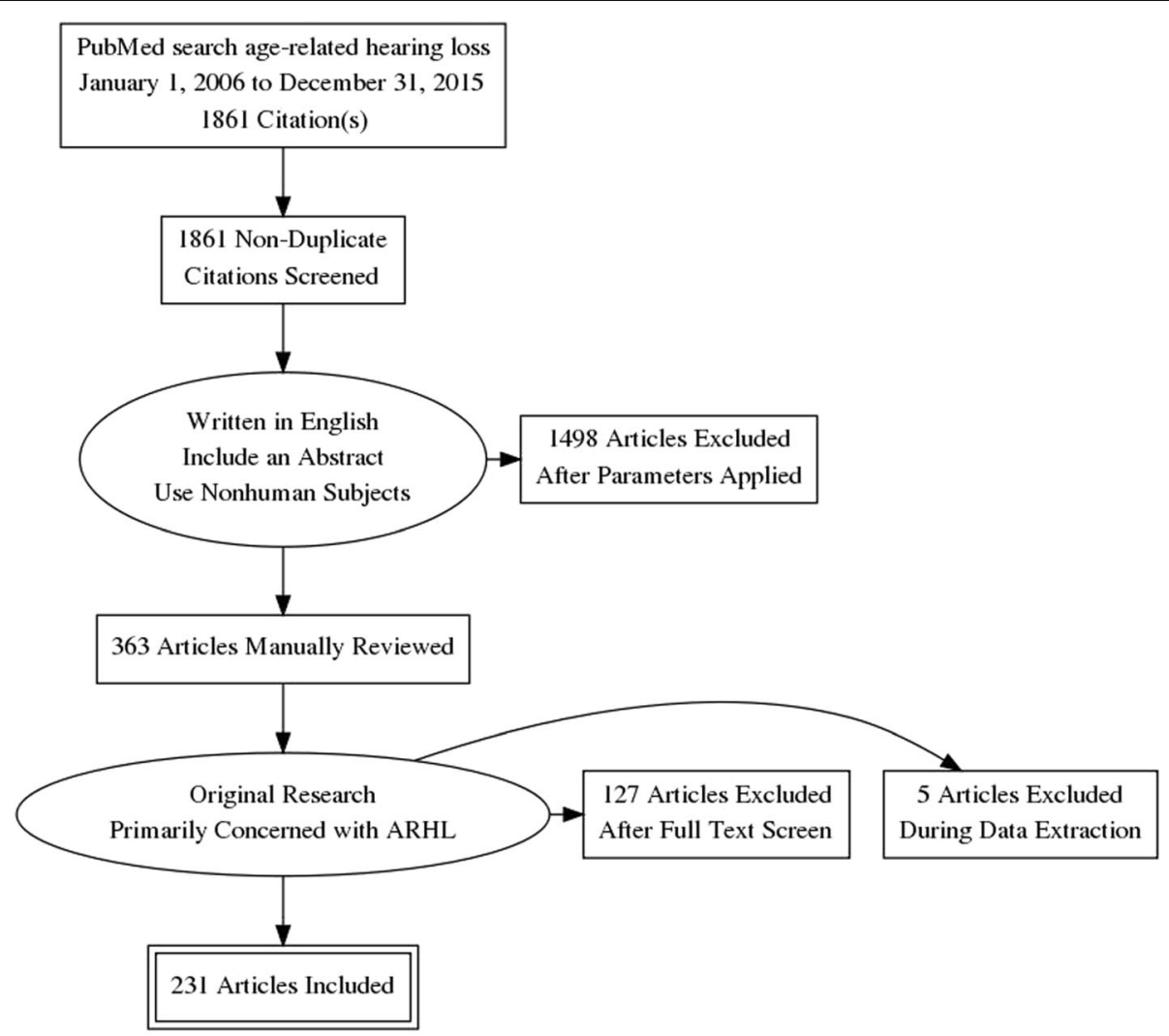

Fig. 1 Flow diagram depicting study review method and inclusion criteria 


\section{Variables coded}

The full text of each manuscript was reviewed for the sex of the animals (males, females, both, or not specified), number of male and female animals, total number of animals, discussion of sex-based results (yes or no), international- or United States (US)-based study, and year of publication.

\section{Data analysis}

We calculated the total number and percentage of manuscripts reporting number of animals used; total number and percentage of manuscripts reporting the sex; total number and percentage of manuscripts using males, females, or both sexes; and total number and percentage of males and females used. We also calculated the total number and percentage of US- and international-based studies, total number and percentage of US and international studies reporting sex, and total number and percentage of international and US-based studies using only males, only females, or both sexes. We also calculated total number and percentage of studies discussing or not discussing sex-based results, total number of international and
US-based studies discussing sex-based results, and percentage of manuscripts discussing sex-based results for the 10-year period.

\section{Results}

\section{Overall sex bias}

Of the total number of manuscripts included, 201 (87\%) reported the total number of animals, while $30(13 \%)$ did not (Fig. 2a). One hundred fifty-two (66\%) manuscripts reported the sex of animals used in the experiments, while 79 (34\%) did not (Fig. 2b). Of these 152 manuscripts reporting sex, 82 (54\%) used both sexes, 51 (34\%) used males only, and 19 (13\%) used females only (Fig. 2c). Of the 154 studies reporting the total number of males (79 studies) and females (46 studies) used, 4125 were males (67\%) and 2077 (33\%) were females (Fig. 2d). The average number of males per study was 52.2 , whereas the average number of females used per study was 45.2 .

\section{Sex bias in US and international studies}

Of the total number of studies, 115 (50\%) were US based and 116 (50\%) were internationally based (Fig. 3a). Of
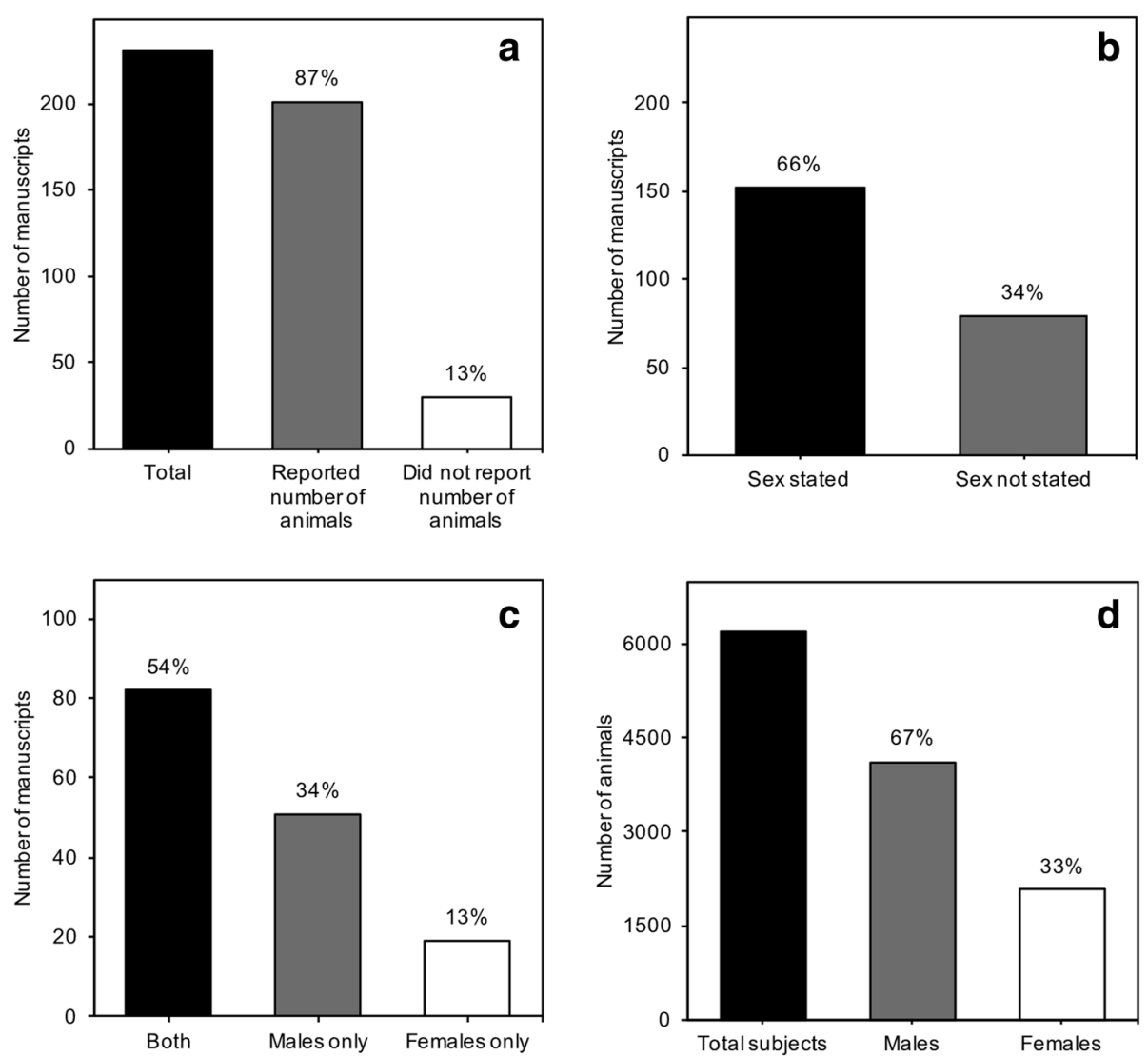

Fig. 2 Overall sex bias in preclinical and basic age-related hearing loss research. a Total number of manuscripts compared to the number of manuscripts reporting and not reporting total number of animals. $\mathbf{b}$ Number of manuscripts specifying the sex and not specifying the sex of animals used in the experiments. c Number of manuscripts using both sexes, males only, or females only. $\mathbf{d}$ Total number of animals in all manuscripts reviewed compared to total number of males and females used 

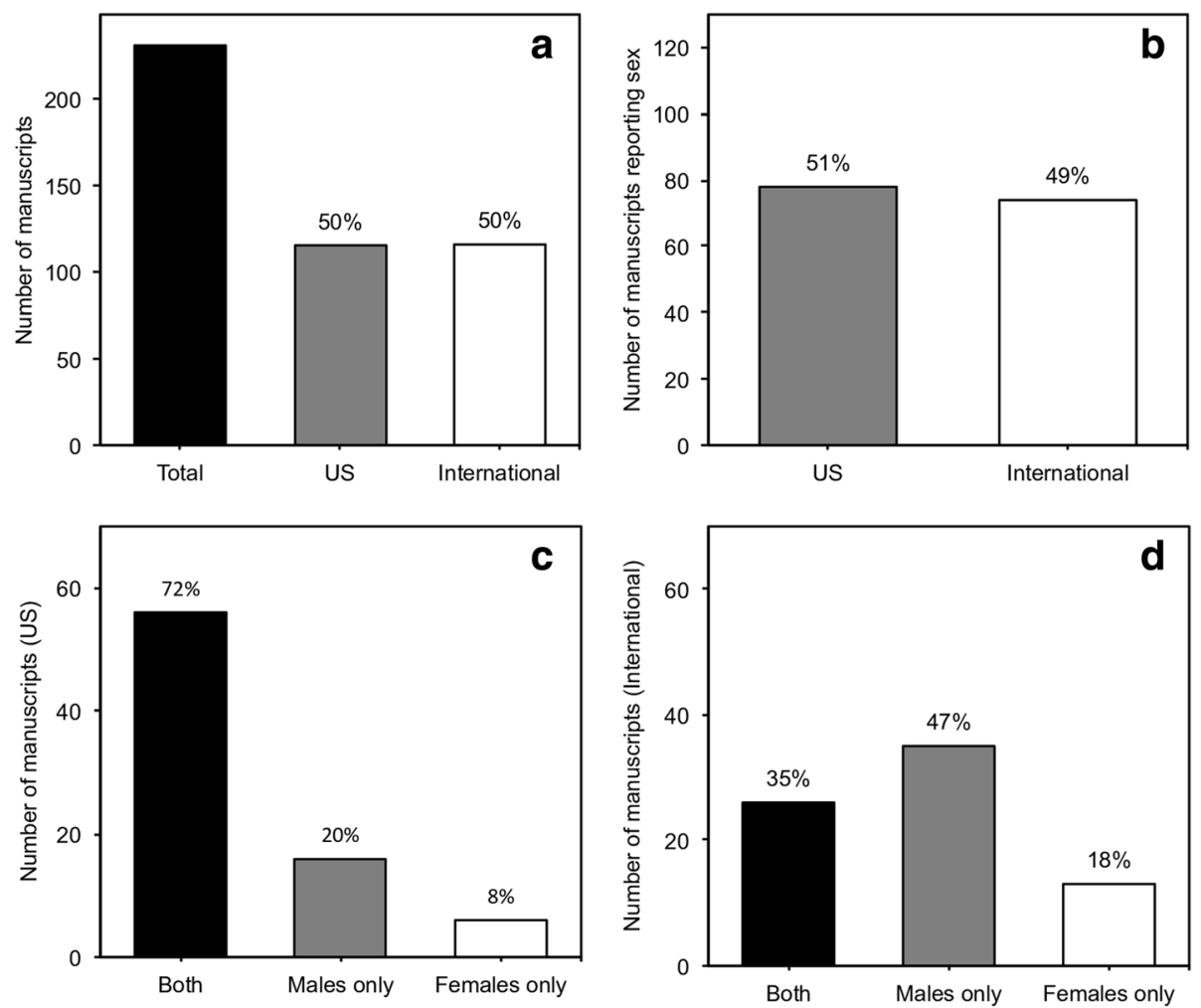

Fig. 3 Sex bias in US and international preclinical and basic age-related hearing loss research. a Total number of studies compared to the number of studies at US- and international-based sites. $\mathbf{b}$ Number of US- and international-based studies reporting sex. $\mathbf{c}$ Number of US-based studies using both sexes, males only, or females only. $\mathbf{d}$ Number of international-based studies using both sexes, males only, or females only

the 152 studies reporting sex, 78 (51\%) were US based and $74(49 \%)$ were internationally based (Fig. 3b). Of the 78 studies reporting sex based in the US, 56 (72\%) used both sexes, $16(20 \%)$ used males only, and $6(8 \%)$ used females only (Fig. 3c). Of the 74 internationally based studies reporting sex, 26 (35\%) used both sexes, 35 (47\%) used males only, and 13 (18\%) used females only (Fig. 3d).

\section{Sex bias over time}

Overall, the number of basic and preclinical manuscripts reporting on ARHL increased from a low of 11 in 2006 to a high of 21 in 2015 (Fig. 4a). The percentage of manuscripts including both males and females fluctuated over the 10-year period, but the highest proportion of studies including both sexes was in 2006 (73\%) and the lowest proportion of studies including both sexes was in
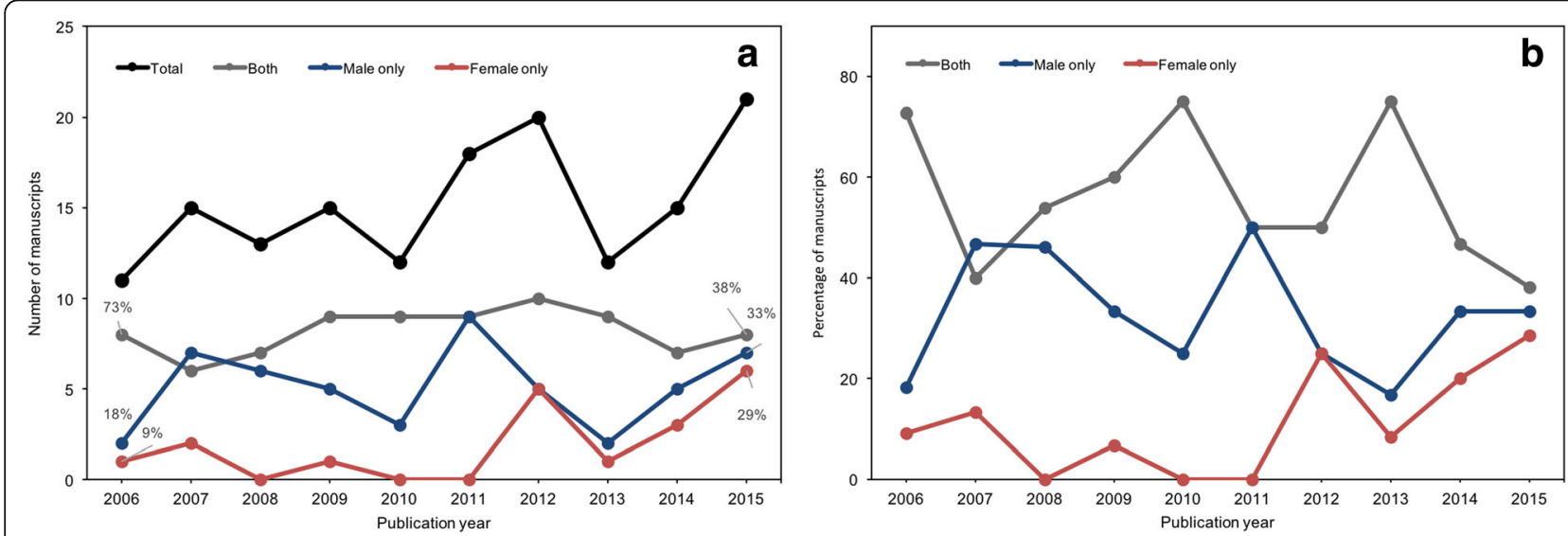

Fig. 4 a Number and $\mathbf{b}$ proportion of manuscripts on basic and preclinical age-related hearing loss using both sexes, males only, or females only from 2006 to 2015 
2015 (38\%) (Fig. 4b). The proportion of studies using males only generally increased over the 10 -year period from 2006 (18\%) to 2015 (33\%). 2007 is the only year where a higher proportion of studies included males only (47\%) than both sexes $(40 \%)$. The proportion of studies using females generally increased from 2006 (9\%) to 2015 (29\%). In no given year did the proportion of studies including only females exceed the proportion of studies using only males or both sexes. In 2008, 2010, and 2011, no studies used females only.

\section{Reporting of sex-based results}

Of the total 231 studies, 34 (15\%) reported sex-based results, while 197 (85\%) did not (Fig. 5a). Of studies reporting sex-based results, 25 (74\%) were US based and 9 (26\%) were internationally based (Fig. 5b). Of the 82 manuscripts using both sexes, 24 (29\%) reported sex-based results. The percentage of manuscripts reporting sex-based results decreased from a high of $54 \%$ in 2006 to a low of $14 \%$ in 2015, although the total number of manuscripts on ARHL increased from 11 in 2006 to 21 in 2015.

\section{Discussion}

This study demonstrates sex bias in basic and preclinical ARHL research. Thirteen percent of studies did not report the total number of animals used. About two thirds of papers reported the sex of animals used, and of studies that reported sex, males only were used over 2.5 times more than females only. For studies that reported the sex and total number of animals used, about two thirds of animals used were male and one third were female. There were a nearly equal number of US-based and international-based studies, with about an equal proportion of US and international studies reporting sex. The proportion of studies including both sexes was over two times greater for US-based studies compared to international-based studies. Five times as many papers did not report sex-based results compared to those that did, and of those that did,
US-based papers were three times more likely to do so than international-based papers.

Over the 10-year period of 2006 to 2015, the proportion of studies using both sexes of animals decreased by $40 \%$, and whereas the proportion of studies including males only increased nearly twofold, the number of females only increased over threefold. Overall, the number of manuscripts on ARHL increased from 2006 to 2015, but there was a nearly $40 \%$ decrease in the number of papers discussing sex-based results. Compared to the previous sex bias study on noise-induced hearing loss (NIHL), this study finds a lower proportion of studies using males only at $34 \%$ (compared to $61 \%$ ) [30]. Additionally, in studies reporting sex and number of animals used, $67 \%$ of all animals used in ARHL studies were males, as opposed to the $81 \%$ found in the study on NIHL [30].

Some investigators cited increased variability related to estrogen cycles to justify the exclusion of females from ARHL studies [31], yet it has been found that the estrous cycle is not generally an influential factor in differences in variability between male and female rodents [32]. When estrous cycle does affect hearing outcomes, studying these effects will improve our understanding of ARHL and could help identify potential new treatments. For example, studying hearing in women and female animals has led to the recognition of estrogen as a possible protective agent against hearing loss [33].

\section{Conclusions}

Sex bias is prevalent in basic and preclinical ARHL research. The inclusion of both males and females in basic and preclinical ARHL research is critical for translational and clinical research outcomes. If only one sex is tested, it is impossible to know which results will generalize to the other sex. By including both sexes in preclinical research, it should be possible to avoid sex-specific issues later in drug development and clinical studies, such as ineffectiveness or
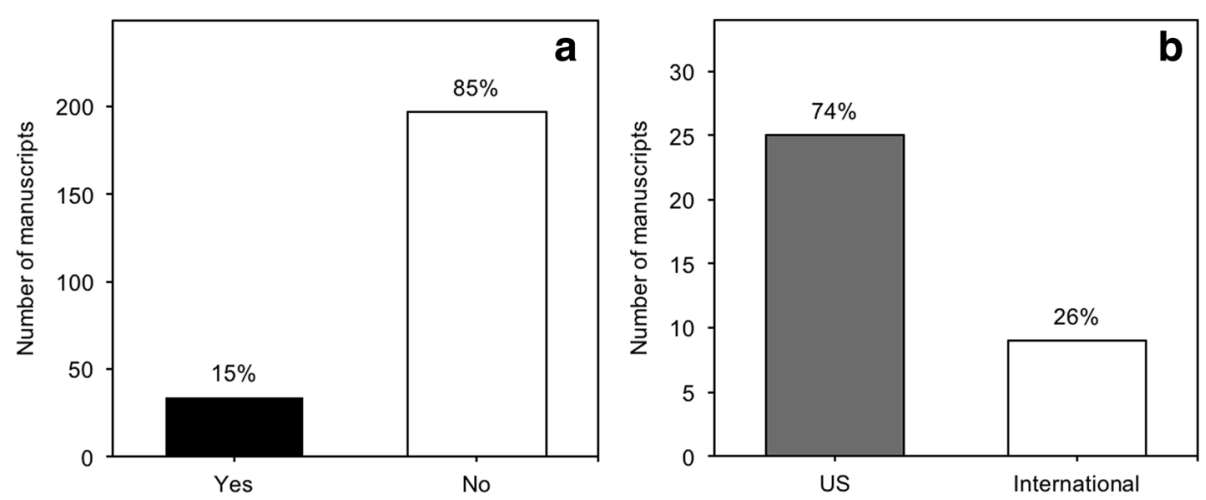

Fig. 5 Reporting of sex-based results in preclinical and basic age-related hearing loss. a Number of studies reporting sex-based results and studies not discussing sex-based results. b Number of US-based and international studies reporting sex-based results 
adverse side effects. When treatments and therapies move to a clinical level, it is important to be able to predict any sex-specific outcomes for better personalization of healthcare treatment. Including sex as a biological variable in basic and preclinical research may reveal other mechanisms of sex differences in ARHL and hasten translation to viable prevention and treatment options.

\section{Abbreviations}

ARHL: Age-related hearing loss; NIHL: Noise-induced hearing loss

\section{Funding}

This study was supported by the David M. Rubinstein Fund for Research to AML.

\section{Availability of data and materials}

The datasets analyzed during the current study are available in the PubMed database.

\section{Authors' contributions}

DFV designed the study, analyzed the data, and wrote the manuscript. AML designed the study and provided critical revision of the manuscript. KMS provided critical revision of the manuscript. All authors read and approved the final manuscript.

\section{Ethics approval and consent to participate}

Not applicable.

\section{Competing interests}

The authors declare that they have no competing interests.

\section{Publisher's Note}

Springer Nature remains neutral with regard to jurisdictional claims in published maps and institutional affiliations.

\section{Received: 20 May 2018 Accepted: 30 May 2018}

\section{Published online: 13 June 2018}

\section{References}

1. Lin FR. Hearing loss in older adults: who's listening? JAMA. 2012;307(11): $1147-8$.

2. Han C, Ding D, Lopez M, Manohar S, Zhang Y, Kim M, et al. Effects of longterm exercise on age-related hearing loss in mice. J Neurosci. 2016;36(44): 11308-19.

3. Lauer AM, Fuchs PA, Ryugo DK, Francis HW. Efferent synapses return to inner hair cells in the aging cochlea. Neurobiol Aging. 2012;33(12):2892-902.

4. Ison J, Allen P, O'Neill W. Age-related hearing loss in C57BL/6J mice has both frequency-specific and non-frequency-specific components that produce a hyperacusis-like exaggeration of the acoustic startle reflex. JARO. 2007;8(4):539-50.

5. Yokoyama JS, Lam ET, Ruhe AL, Erdman CA, Robertson KR, Webb AA, et al. Variation in genes related to cochlear biology is strongly associated with adult-onset deafness in border collies. PLoS Genet. 2012;8(9):e1002898.

6. Lin FR, Yaffe K, Xia J, Xue Q, Harris TB, Purchase-Helzner E, et al. Hearing loss and cognitive decline in older adults. JAMA Intern Med. 2013;173(4):293-9.

7. Mick P, Kawachi I, Lin FR. The association between hearing loss and social isolation in older adults. Otolaryngol Head Neck Surg. 2014;150(3):378-84.

8. Rabbitt P. Mild hearing loss can cause apparent memory failures which increase with age and reduce with IQ. Acta Otolaryngol. 1991;111(\$476): $167-76$.

9. Lin FR, Ferrucci L. Hearing loss and falls among older adults in the United States. Arch Intern Med. 2012;172(4):369-71.

10. Genther DJ, Frick KD, Chen D, Betz J, Lin FR. Association of hearing loss with hospitalization and burden of disease in older adults. JAMA. 2013;309(22): 2322-4.

11. Genther DJ, Betz J, Pratt S, Kritchevsky SB, Martin KR, Harris TB, et al. Association of hearing impairment and mortality in older adults. J Gerontol A Biol Sci Med Sci. 2015;70(1):85-90.
12. Kim S, Lim EJ, Kim HS, Park JH, Jarng SS, Lee SH. Sex differences in a cross sectional study of age-related hearing loss in Korean. Clin Exp Otorhinolaryngol. 2010;3(1):27-31.

13. Pearson JD, Morrell $\mathrm{CH}$, Gordon-Salant $\mathrm{S}$, Brant $\mathrm{L}$, Metter EJ, Klein $\mathrm{LL}$, et al. Gender differences in a longitudinal study of age-associated hearing loss. J Acoust Soc Am. 1995;97(2):1196-205.

14. Turner JG, Parrish JL, Zuiderveld L, Darr S, Hughes LF, Caspary DM, et al. Acoustic experience alters the aged auditory system. Ear Hear. 2013;34(2): 151-9.

15. Willott JF, Turner JG, Sundin VS. Effects of exposure to an augmented acoustic environment on auditory function in mice: roles of hearing loss and age during treatment. Hear Res. 2000;142(1):79-88.

16. Willott JF. Effects of sex, gonadal hormones, and augmented acoustic environments on sensorineural hearing loss and the central auditory system: insights from research on C57BL/6J mice. Hear Res. 2009;252(1-2): 89-99.

17. Konig O, Ruttiger L, Muller M, Zimmermann U, Erdmann B, Kalbacher H, et al. Estrogen and the inner ear: megalin knockout mice suffer progressive hearing loss. FASEB J. 2008:22(2):410-7.

18. Guimaraes P, Zhu X, Cannon T, Kim S, Frisina RD. Sex differences in distortion product otoacoustic emissions as a function of age in CBA mice. Hear Res. 2004:192(1):83-9.

19. Henry KR. Sex- and age-related elevation of cochlear nerve envelope response (CNER) and auditory brainstem response (ABR) thresholds in C57BL/6 mice. Hear Res. 2002;170(1-2):107-15.

20. Willott JF, VandenBosche J, Shimizu T, Ding D, Salvi R. Effects of exposing C57BL/6J mice to high- and low-frequency augmented acoustic environments: auditory brainstem response thresholds, cytocochleograms, anterior cochlear nucleus morphology and the role of gonadal hormones. Hear Res. 2008:235(1-2):60-71.

21. Rubio DM, Schoenbaum EE, Lee LS, Schteingart DE, Marantz PR, Anderson $\mathrm{KE}$, et al. Defining translational research: implications for training. Acad Med. 2010;85(3):470-5.

22. Clayton JA, Collins FS. Policy: NIH to balance sex in cell and animal studies. Nature News. 2014;509(7500):282

23. Galkina Cleary E, Beierlein JM, Khanuja NS, McNamee LM, Ledley FD. Contribution of NIH funding to new drug approvals 2010-2016. Proc Natl Acad Sci U S A. 2018;115(10):2329-34.

24. Karp NA, Mason J, Beaudet AL, Benjamini Y, Bower L, Braun RE, et al. Prevalence of sexual dimorphism in mammalian phenotypic traits. Nat Commun. 2017:8:15475.

25. Kong BY, Haugh IM, Schlosser BJ, Getsios S, Paller AS. Mind the gap: sex bias in basic skin research. J Invest Dermatol. 2016;136(1):12-4.

26. Ramirez FD, Motazedian P, Jung RG, Di Santo P, MacDonald Z, Simard T, et al. Sex bias is increasingly prevalent in preclinical cardiovascular research: implications for translational medicine and health equity for women: a systematic assessment of leading cardiovascular journals over a 10-year period. Circulation. 2017;135(6):625-6.

27. Yoon DY, Mansukhani NA, Stubbs VC, Helenowski IB, Woodruff TK, Kibbe MR. Sex bias exists in basic science and translational surgical research. Surgery. 2014;156(3):508-16.

28. Will TR, Proaño SB, Thomas AM, Kunz LM, Thompson KC, Ginnari LA, et al. Problems and progress regarding sex bias and omission in neuroscience research. eNeuro. 2017:4(6)

29. Beery AK, Zucker I. Sex bias in neuroscience and biomedical research. Neurosci Biobehav Rev. 2011;35(3):565-72.

30. Lauer AM, Schrode KM. Sex bias in basic and preclinical noise-induced hearing loss research. Noise Health. 2017;19(90):207-12.

31. Vasilyeva ON, Frisina RD, Frisina ST, Zhu X, Walton JP. Interactions of hearing loss and diabetes mellitus in the middle age CBA/CaJ mouse model of presbycusis. Hear Res. 2009;249(1):44-53.

32. Prendergast BJ, Onishi KG, Zucker I. Female mice liberated for inclusion in neuroscience and biomedical research. Neuroscience \& Biobehavioral Reviews. 2014 March 1:40:1-5.

33. Hultcrantz $M$, Simonoska R, Stenberg AE. Estrogen and hearing: a summary of recent investigations. Acta Otolaryngol. 2006;126(1):10-4. 